Slavica

bruxellensia

\section{Slavica bruxellensia}

Revue polyphonique de littérature, culture et histoire

slaves

6 | 2010

Linguistique russe

\title{
Esthétique et théorie du roman : la théorie dialogique du Bakhtine linguiste
}

\section{Laura Calabrese-Steimberg}

\section{OpenEdition}

Journals

Édition électronique

URL : http://journals.openedition.org/slavica/348

DOI : 10.4000/slavica.348

ISSN : 2034-6395

Éditeur

Université libre de Bruxelles - ULB

Édition imprimée

Pagination : 60-64

ISSN : 2031-7654

Référence électronique

Laura Calabrese-Steimberg, "Esthétique et théorie du roman : la théorie dialogique du Bakhtine linguiste », Slavica bruxellensia [En ligne], 6 | 2010, mis en ligne le 15 juin 2010, consulté le 19 avril 2019. URL : http://journals.openedition.org/slavica/348; DOI : 10.4000/slavica.348

Ce document a été généré automatiquement le 19 avril 2019

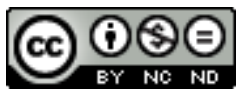

Les contenus de Slavica bruxellensia sont mis à disposition selon les termes de la Licence Creative Commons Attribution - Pas d'Utilisation Commerciale - Pas de Modification 3.0 France. 


\title{
Esthétique et théorie du roman : la théorie dialogique du Bakhtine linguiste
}

\author{
Laura Calabrese-Steimberg
}

1 L'œuvre de Mikhaïl Bakhtine (1895-1975)occupe, depuis les années 1960, une place fondamentale dans l'analyse littéraire. Elle est lue et commentée dans les universités d'Europe, d'Amérique du Nord et d'Amérique latine. Or, cette œuvre est également centrale dans le domaine des sciences du langage, et tout particulièrement de la linguistique du discours, depuis qu'elle a été introduite dans le champ français par Tzvetan Todorov et Julia Kristeva. Cette introduction des textes de Bakhtine n'a pas été sans difficulté, étant donné l'obstacle de la langue (peu d'intellectuels francophones connaissant le russe), mais elle a été accompagnée d'une impressionnante force d'imprégnation, pénétrant la totalité du champ des sciences humaines, comme le note l'analyste du discours Jean Peytard ${ }^{1}$. Cependant, suite à l'appropriation très personnelle qu'en font les deux intellectuels bulgares, le Bakhtine français est perçu comme "l'initiateur de la théorie de l'énonciation, sorte d'élève de [Émile] Benveniste avant l'heure, ou bien un rénovateur de la théorie marxiste des idéologies $»^{2}$. En effet, lorsque son œuvre est traduite durant les années 1970, elle provoque un effet de réception singulier dans les cercles intellectuels français ${ }^{3}$, qui font du théoricien russe un précurseur des théories du discours. Trente ans plus tard, les retours théoriques et épistémologiques sur l'œuvre de Bakhtine permettent de la replacer dans son contexte de production et de faire un bilan de son incalculable apport aux sciences du langage.

2 Les concepts bakhtiniens qui ont le plus marqué ce domaine disciplinaire sont certainement ceux de "polyphonie »etde "dialogisme ", et ce malgré leurs fluctuations de sens, qui ont donné lieu à une réflexion épistémologique importante et à des débats quasi philologiques en raison de l'inaccessibilité des textes originaux pour la plupart des linguistes occidentaux. Comme le note Aleksandra Nowakowska, «son vocabulaire est complexe et difficile à cerner, donc à traduire, car l'auteur, soit utilise des termes existants (dialogichnost', raznorechie), soit fait un emploi particulier, souvent étendu, des termes existants (dialogicheskij) $»^{4}$. Une difficulté majeure réside dans le fait que les deux 
notions se recoupent, désignant plus largement l'hétérogénéité des voix et de points de vue propre à tout énoncé.

3 Le concept de polyphonie (polifonija), développé dans l'ouvrage Problèmes de la poétique de Dostoïevski, est d'emblée associé à la construction romanesque, pour décrire notamment l'œuvre de l'auteur russe. Si la notion est proposée dès 1929, elle n'est pas reprise par la suite. Au contraire, la notion de «dialogisme», notammentprésentée dans Esthétique et théorie du roman et Esthétique de la création verbale, parcourt toute son œuvre. La pragmatique, via les travaux d'Oswald Ducrot ${ }^{5}$, exploite le concept de « polyphonie » dans le cadre d'une linguistique énonciative. Or, Ducrot déplace le champ d'application de la polyphonie du texte vers l'énoncé, dans lequel il distingue la voix du sujet parlant (être empirique) de celles du locuteur et de l'énonciateur (être de discours). Ces trois entités peuvent être assimilées aux notions littéraires "d'auteur", "narrateur » et «personnage ». Cette modélisation lui permet d'analyser les cas de double énonciation au sein d'un même énoncé, en termes de responsabilité énonciative et de point de vue. Le cas de la négation en est un exemple typique. Si j'affirme que «mon bureau ne se trouve pas loin du centre ", je (locuteur) mets en scène un énonciateur qui suppose que mon bureau se trouve loin du centre, dont je fais entendre la voix dans le même énoncé.

Malgré la dette (partiellement assumée par Ducrot) envers le théoricien, ses développements sont loin du projet bakhtinien de lier un énoncé à un réseau d'énoncés antérieurs et futurs (voir infra), c'est-à-dire à son extérieur, et se limite à l'énoncé phrastique ${ }^{6}$. Malgré cela, nul ne doute de la place stable qu'occupe le concept de "polyphonie" en linguistique énonciative, qui s'intègre dans une série plus large de phénomènes discursifs tels que l'ironie, le pastiche et la parodie. L'incorporation du concept à la pragmatique énonciative a par ailleurs donné lieu à toute une école d'analyse polyphonique, la ScaPoLine (théorie SCAndinave de POlyphonie LINguistiquE), laquelle envisage autant l'énoncé que le texte comme une mise en scène de plusieurs voix. Les polyphonistes scandinaves (qui, contrairement à Bakhtine, conçoivent la coexistence de voix selon un modèle hiérarchique), considèrent la polyphonie comme un principe sémantique qui guide l'interprétation de l'énoncé.

5 Contrairement à la polyphonie, qui constitue un principe de la construction romanesque, le « dialogisme » (tel qu'il a été traduit en français) est un principe qui domine la pratique langagière, car tout énoncé est pris dans un réseau d'interactions, et ce, dans deux sens au moins. D'une part, tout énoncé est en interaction avec d'autres énoncés, car notre discours rencontre les discours antérieurs qui ont été produits par rapport à ce même sujet. Puisque tous les mots ont déjà existé dans la bouche d'un autre énonciateur, nous parlons avec les mots des autres, car « il n'existe plus, depuis Adam, d'objets innommés, ni de mots qui n'auraient pas déjà servi $»^{7}$. D'autre part, chaque prise de parole se fait en fonction de notre interlocuteur (in praesentia ou in absentia). Autrement dit, chaque fois que nous prenons la parole nous sommes, en quelque sorte, en situation de dialogue : «Se constituant dans l'atmosphère du "déjà dit", le discours est déterminé en même temps par la réplique non encore dite, mais sollicitée et déjà prévue $»^{8}$. Cela revient à dire que le discours est radicalement hétérogène car il est en permanence traversé par l'altérité. En effet, les développements bakhtiniens remettent en cause non seulement l'unicité de l'énoncé, mais également celle du sujet parlant.

On comprend bien le rôle que joue l'œuvre de Bakhtine dans le détrônement du structuralisme français, qui se focalise sur l'analyse de l'énoncé au détriment de l'énonciation et des sujets énonciateurs. Le principe dialogique, au contraire, ouvre le 
texte vers son extériorité et son antériorité, car comment analyser un énoncé sans prendre en compte la situation d'énonciation et les énoncés antérieurs avec lesquels il entre relation? L'énoncé bakhtinien est ainsi «une unité de la chaîne verbale ininterrompue $»^{9}$, traversée, qui plus est, par les voix de ceux qui ont employé les mêmes mots avant: "Chaque mot sent la profession, le genre, le courant, le parti, l'œuvre particulière, l'homme particulier, la génération, l'âge et le jour. Chaque mot sent le contexte et les contextes dans lesquels il a vécu sa vie sociale intense... $»^{10}$.

7 Le principe dialogique introduit non seulement une nouvelle approche du fait langagier, mais également du sujet parlant. Dans le modèle bakhtinien, la parole n'est pas un lieu d'émergence de l'individualité mais, bien au contraire, la manifestation d'une activité collective qui témoigne de l'insertion de chacun dans le thésaurus commun du langage. Il dépasse par là l'approche stylistique, pour laquelle les textes sont l'expression d'un individu. À la stylistique et la linguistique structurelle, le théoricien oppose un modèle éclaté, multiple, hétéroclite des énoncés et des énonciateurs, selon lequel le sujet n'est pas la source unique du sens, qu'il partage avec ses interlocuteurs et, plus largement, avec toute la communauté langagière.

Ce virage épistémique opéré par son œuvre dans le champ français (et francophone) va profiter tout particulièrement à l'analyse de discours naissante (fin des années 1960), parfois au détriment d'une discrimination claire des concepts. L'analyse de discours française $\left(\mathrm{ADF}^{11}\right)$ va s'atteler à repérer des marques concrètes du dialogisme qui se trouve à la base de tout acte de langage ; soucieux de faire parler les discours au-delà de ce que les énonciateurs disent, les analystes du discours vont chercher des traces d'altérité dans tout énoncé, dans ses échos, ses résonances. Il faut par ailleurs rappeler que l'idée d'un principe dialogique s'adapte tout naturellement au concept d'interdiscours de l'ADF, avec lequel il se confond souvent, et que les théoriciens érigent également en principe de tout discours ${ }^{12}$. Cependant, le concept de Bakhtine, en suggérant la présence de plusieurs voix et la tenue d'un dialogue in-absentia, garde une force métaphorique que l'interdiscours n'a pas. Il s'insère par ailleurs dans le cadre plus large d'une théorie du sujet pluriel que l'ADF fera sienne très tôt dans l'évolution de la discipline.

9 C'est ainsi dans le sillage de la théorie dialogique que Jacqueline Authier développera les concepts « d'hétérogénéité montrée » et "d'hétérogénéité constitutive » ${ }^{13}$, un pilier de l'ADF, qui soutient que la plupart des discours sont traversés par des discours autres, que ce soit explicitement ou implicitement. Parmi les formes marquées ou explicites, on compte le discours direct ou indirect, les guillemets ou les gloses, qui signalent une distance entre le locuteur et son énoncé («Comment dirais-je?», «Passez-moi l'expression », "Ce n'est pas le mot qui convient »). Le discours indirect libre, l'allusion, l'ironie ou le pastiche ${ }^{14}$ font partie des formes non marquées et peuvent être (ou non) identifiés par l'interlocuteur en fonction de ses connaissances encyclopédiques. Pour sa part, l'hétérogénéité constitutive est un principe de construction de tout discours, traversé comme il est par l'altérité (y compris l'altérité du sujet parlant, qui peut se dédoubler pour gloser son propre énoncé), pris dans un dialogue permanent avec l'Autre. Le modèle d'Authier, largement travaillée par la psychanalyse lacanienne, rejoint ainsi la vision bakhtinienne, qui remet en cause l'unicité du sujet parlant, et ouvre la voie à une sémantique des discours soucieuse de la construction collective du sens.

Dans le domaine du discours médiatique, les travaux de Sophie Moirand déconstruisent le parcours mémoriel de certaines désignations médiatiques, construites sur des échos de désignations antérieures. S'inscrivant dans la tradition du dialogisme bakhtinien, 
Moirand part à la recherche de séquences linguistiques capables d'évoquer, de suggérer ou de représenter des paroles antérieures, sans qu'elles soient explicitement marquées. Les exemples suivants laissent entendre des échos de dires antérieurs :

11 Dans ces exemples, il y aurait « comme quelque chose qu'on aurait déjà entendu [...] qui peut être de l'ordre du "vague", et qui serait stocké dans la mémoire du lecteur, sans quoi l'allusion ne peut être "sentie" et reste sans "effet" : mémorisation antérieure de formes, de sons, de sens, et même de constructions $»^{18}$. Les travaux de Moirand montrent, à travers l'observation du principe dialogique dans les discours sociaux, un mécanisme fondamental de ceux-ci (et notamment du discours médiatique), à savoir, la création de domaines de mémoire partagés ou faits de mémoire collectifs, qui façonnent notre perception des événements médiatiques et, plus largement, du fait public. L'étiquette métalinguistique de dialogisme interdiscursif, avec laquelle Moirand désigne le phénomène, montre à quel point les concepts de dialogisme et d'interdiscours se recoupent.

Si des imprécisions conceptuelles demeurent dans l'interprétation francophone du théoricien russophone (certes, de moins en moins importantes, grâce au travail mené par plusieurs spécialistes dans le domaine de la traductologie et de la critique génétique ${ }^{19}$ ), son travail a permis de préciser une série de mécanismes à l'œuvre dans les productions langagières, fondamentaux pour comprendre les discours publics, la mémoire collective, la forme sociale du langage et la configuration hétérogène du sujet parlant. Son œuvre, rédigé en Union Soviétique entre la fin des années 1920 et les années 1960, rencontre ainsi, par le biais d'un dialogie différé, celle de plusieurs théoriciens européens majeurs, tels qu'Émile Benveniste, Maurice Halbwachs, Louis Altusser ou Jacques Lacan, dont les travaux ont été également décisifs pour le développement d'une linguistique du discours.

\section{NOTES}

1. Peytard J., Mikhail Bakhtine. Dialogisme et analyse du discours, Bertrand-Lacoste, Paris, 1995, $128 \mathrm{p}$.

2. Sériot cité in : Agueeva I., «Le M. Bakhtine "français" : la réception de son œuvre dans les années 1970 », in : http://cid.ens-lsh.fr/russe/lj_agueeva.htm. Consulté le 28 juin 2010.

3. Voir par exemple Peytard J., Op. cit. \& Agueeva I., Art. cit.

4. Nowakowska A., «Dialogisme et polyphonie : des textes russes de M. Bakhtine à la linguistique contemporaine ", in : Dialogisme et polyphonie. Approches linguistiques (Sous la direction de Bres J. et alii), De Boeck-Duculot, Bruxelles, 2005, p. 25.

5. Le dire et le dit, Paris, Minuit, 1984.

6. Bres J. \& Rosier L., «Réfractions : polyphonie et dialogisme, deux exemples de reconfigurations théoriques dans les sciences du langage francophones ", in : "SLAVICA OCCITANIA ", n 25, 2007, pp. 437-461.

7. Todorov Ts., Mikhail Bakhtine, le principe dialogique, Le Seuil, Paris, p. 8.

8. Bakhtine M., Esthétique et théorie du roman, traduit du russe par Daria Olivier, Gallimard, Paris, p. 103.

9. Zavialoff N., "L'énonciation chez Bakhtine, une explication restrictive », in: Depretto C., L'héritage de Mikhail Bakhtine, Presse Universitaires de Bordeaux, Bordeaux, 1997. 
10. Todorov Ts., Op. Cit., p. 89.

11. L'Analyse du discours française, qui surgit à la fin des années 1960 , se donne pour objet d'étude des énoncés - organisés en corpus - situés dans leurs conditions de production historiques et politiques (et non des phrases). Cette observation doit permettre de déduire la conformation idéologique d'un discours.

12. «Tout discours est traversé par de l'interdiscursivité, il a pour propriété constitutive d'être en relation multiforme avec d'autres discours, d'entrer dans l'interdiscours. Ce dernier est au discours ce que l'intertexte est au texte" (Charaudeau P. \& Maingueneau D., Dictionnaire de l'analyse du discours, Paris, Le Seuil, 2002, p. 24).

13. Voir Authier-Revuz J., «Hétérogénéité montrée et hétérogénéité constitutive : éléments pour une approche de l'autre dans le discours ", in : «DRLAV », $n^{\circ} 26,91-151$ et Ces mots qui ne vont pas de soi. Boucles réflexives et non-cö̈ncidences du dire, 2 t. Larousse, coll. « Science du langage », Paris, 1995, $689 \mathrm{p}$.

14. Voir Charaudeau P. \& Maingueneau D., Dictionnaire de l'analyse du discours, Le Seuil, Paris, 2002, $666 \mathrm{p}$.

15. Tiré d'un article du journal «LE MONDE» du 15 août 1999 (allusion au livre de V. Forrester, L'horreur économique).

16. Tiré d'un article du journal «LE MONDE» du 27 mai 2000 (allusion aux paroles de la Marseillaise).

17. Tiré d'un article du journal «LE MONDE» du 25 juin 2003 (allusion au discours de Bush sur la «croisade contre l'axe du mal », après le 11 septembre 2001, lequel fait à son tour allusion aux croisades du Moyen Âge).

18. Moirand S., "Discours, mémoires et contextes: à propos du fonctionnement de l'allusion dans la presse », Corela, Cognition, discours, contextes. http://edel.univ-poitiers.fr/corela/ document.php?id=1636. Consulté le 28 juin 2010.

19. Voir Nowakowska A., Art. cit. et Bres J. \& Rosier L., Art. cit. mais également Vauthier B., "Mikhail Bakhtine, Valentin Volochinov et Pavel Medvedev dans les contextes européen et russe », in : «SLAVICA OCCITANIA », $\mathrm{n}^{\circ} 25,2007$.

\section{INDEX}

Index géographique : Russie

Mots-clés : analyse littéraire, linguistique russe, théorie de la littérature

\section{AUTEURS}

\section{LAURA CALABRESE-STEIMBERG}

Membre de l'équipe Ladisco de l'Université Libre de Bruxelles 